\title{
Epistemología, axiología y aprendizaje de la ciencia en el aula
}

\author{
Epistemology, axiology and learning of science in the classroom
}

Maria Rosa Simonelli De Yaciofano

Universidad Pedagógica Experimental Libertador. $\quad$ mr simonelli@yahoo.com
mr2 simonelli@hotmail.com

\section{Resumen}

Con el propósito de estudiar la interacción en pedagogía, ciencia y metaciencia, se da a conocer, el estudio epistemológico, que se presenta en dos planos; el primero plano se ubica en la presentación de una base documental de la enseñanza-aprendizaje, en el enfoque positivista y conductista en la producción del conocimiento desde el siglo XIX, XX y XXI. El segundo plano epistemológico, al aula como ámbito espacial y centro de acción de los procesos actuales que allí viven los docentes y los discentes, incluye el tema de la ciencia desde los modos de impartir y producir el conocimiento en el proceso de enseñanza-aprendizaje; como en lo que se refiere a la concepción que de ella y su producto se moviliza desde diversas instancias de la dinámica social, induyendo la educación, con el objetivo de contribuir con el tendido del puente epistemología axiología - aprendizaje de la ciencia. El desarrollo de este estudio, se da a conocer investigaciones de diferentes autores que canalizan la preocupación detectada y el estudio del pensamiento pedagógico y metacient́fico de profesores de la enseñanza de la didáctica y de la ciencias; ayos hallazgos nos aproximan a las verdades provisionales, como un intento de resolver el vacio teórico o epistemológico del trabajo docente - aprendizaje - metaciencia y producción del conocimiento de la ciencia, tiene como propósito dar a conocer la interacción pedagogía, ciencia y metaciencia; considerando el aula el ámbito espacial y centro de acción de los procesos que allí viven los docentes y estudiantes e induye el tema de la ciencia y la concepción que de ella se tiene, a nivel de educación superior. Para ello se emplean lineamientos teóricos cognitivos, conductistas y constructivistas, a fin de develar la estructura y la dinámica en un espacio para el aprendizaje. El estudio se abordará bajo el enfoque cualitativo de una investigación fenomenológica en la interacción docente-discente en el aula; se enmarca en una metodología de tipo descriptiva-documental. Se propone con el estudio el desarrollo, de un modelo educativo socio cognitivo, producto de una investigación cualitativa de la dinámica de aula, entre la epistemología - axiología - aprendizaje, centrado en un paradigma de desarrollo integral, sobre la base de una didáctica científico-cática.

\section{Palabras clave}

Axiología, metaciencia, ciencia.

\section{Abstract}

In order to study the interaction in education, science and metaciencia, is known, the epistemological study, presented at two levels: the first plane is in the presentation of an evidence base of teaching and leaming in positivist and behaviourist approach in the production of knowledge from the century XIX, XX and XXI. The second epistemological level, the dassroom space and action center of the current processes that inhabit the teachers and learners, induding the issue of science from the modes of producing and delivering knowledge in the teaching-leaming process; in regard to the design of it and its product be mobilized from various levels of social dynamics, induding education, to help with the laying of the bridge epistemology - axiology - leaming of science. The development of this study, research reveals that different authors channeled the concems identified and the study of pedagogical thinking and metacientifico teacher education and the teaching of 
science, whose findings we are dose to the provisional truths, such as an attempt to resolve the theoretical and epistemological vacuum of teaching - leaming - metaciencia and knowledge production in science, aims to raise awareness of the interaction pedagogy, science and metaciencia; considering dassroom space and the center of the action processes that inhabit the teachers and students and indudes the topic of science and the conception that it is at the level of higher education. This theoretical guidelines used cognitive, behavioral and constructivist, to reveal the structure and dynamics in a space for leaming. The study will be addressed under the qualitative approach of a phenomenological investigation into the teacher-pupil interaction in the dassroom is part of a methodology for desaiptive-documentary. It is proposed to study the development of a social cognitive model of education, the product of a qualitative study of the dynamics of the dassroom, between epistemology - axiology - learning-centered paradigm of development based on a scientificdidactic critical.

\section{Keywords}

Axiology, metascience, science.

\section{Introducción}

La situación problemática abordada en esta investigación tiene como propósito dar a conocer una amplia esfera de interacción en pedagogía, ciencia y metaciencia; la cual se presenta en dos planos epistemológicos del fenómeno estudiado. El primero de ello se encuentra relacionado con una base documental de investigaciones realizadas desde la perspectiva en la cual se venía enfocando la enseñanza-aprendizaje del conocimiento basado en el método científico la ciencia; desde el siglo XIX, XX y XXI.

El segundo plano epistemológico se corresponde, al aula como ámbito espacial y centro de acción de los procesos actuales que allí viven los docentes y los discentes, induye el tema de la ciencia desde los modos de impartir y producir el conocimiento en el proceso de enseñanzaaprendizaje; como en lo que se refiere a la concepción que de ella y su producto se moviliza desde diversas instancias de la dinámica social, induyendo la educación, con el objetivo de contribuir con el tendido del puente epistemología - axiología - aprendizaje de la ciencia. Además de renovar la perspectiva en auanto a los enfoques de enseñanza-aprendizaje, que pone en juego el docente, con el fin de mejorar la didácica de enseñanza y el aprendizaje; se busca con preocupación que, los estudiantes en lugar de recibir, pasivamente como creía ingenuamente la vieja pedagogía, llegan a construir los conceptos científicos en una forma significativa.

La principal orientación teórica es dar a conocer mediante estudios científicos, una serie de referentes sobre el quehacer educativo del saber científico desde los enfoques conductista y constructivista de la ciencia; basado en una metodología documental, que obedece al compromiso de estudiar la misma, en un paradigma cualitativo, fenomenológico y de carácter naturalista.

En el desarrollo de este estudio, se da a conocer investigaciones de diferentes autores que canalizan la preocupación detectada y el estudio del pensamiento pedagógico y metacient́fico de profesores de la enseñanza de la didáctica y de la ciencias; ayos hallazgos nos aproximan a las verdades provisionales, como un intento de resolver el vacío teórico o epistemológico del trabajo docente - aprendizaje - metaciencia. Así mismo, se propone con dicha investigación, la ideación y construcción de un modelo de enseñanza de enfoque constructivista; que desartiaulen, los hitos más importantes del proceso de enseñanza-aprendizaje de un concepto científico y el conjunto de relaciones que se suscitan entre las entidades del modelo: alumno, dase, docente, siendo el aula un medio complejo. 


\section{Enseñanza tradicional}

La enseñanza transmisiva tradicional, aún hoy mayoritaria en el plano educativo de nuestro país, constituye una herencia cultural de los siglos XIX y XX, resulta sin duda, un modelo educativo que no puede satisfacer los requerimientos de la sociedad actual. Las sociedades actuales, caracterizadas por los procesos de globalización, flexibilidad e interculturabilidad, parecen requerir otros tipos de enfoques educativos que generen nuevas capacidades y conocimientos para afrontar la incertidumbre y para promover la comprensión y el tratamiento de problemas en el campo de la didáctica educativa.

En tal sentido, al realizar un análisis diacrónico en la historia de impartir el conocimiento y nos ubicamos en épocas pasadas; encontramos que uno de los métodos que se mantiene con gran relevancia y prestigio es el "método científico", argumentado bajo el pensamiento de Augusto Comte (1798-1857) y de Emilio Durkheim (1858-1917), ambos citados por Ander-Egg, (1993) p.12.

En 1962, Kuhn publicó La estructura de las revoluciones científicas, en donde exponía la evolución de las ciencias naturales básicas de un modo que se diferenciaba la forma sustancial de la visón más generalizada entonces. Según Kuhn, las ciencias no progresan siguiendo un proceso uniforme por la aplicación de un hipotético método cient́fico. Se verifican, en cambio, dos fases diferentes de desarrollo cientúfico. En un primer momento, hay un amplio consenso en la comunidad científica sobre cómo explotar los avances conseguidos en el pasado ante los problemas existentes, creándose así soluciones universales que Kuhn llamaba "paradigmas".

En un segundo momento, se buscan nuevas teorías y herramientas de investigación conforme las anteriores dejan de funcionar con eficacia. Si se demuestra que una teoría es superior a las existentes entonces es aceptada y se produce una "revolución científica". Tales rupturas revolucionarias traen consigo un cambio de conceptos cient́ficos, problemas, soluciones y métodos, es decir, nuevos "paradigmas". Siendo este término desarrollado por Kuhn (2004) a comienzos de la década de los 60, presentado en el libro de las "la estructura de las revoluciones científicas", definiéndolo como realizaciones cient́ficas universalmente reconocidas que, durante cierto tiempo, proporcionaron modelos de problemas y soluciones a una comunidad científica.

Es entones, cuando las actividades basadas en el método científico, se basan en los supuestos y postulados del paradigma; se plantean enigmas (problemas) y se establece el camino válido para sus posibles soluciones, se definen los intereses de la ciencia. Luego de un largo camino de investigaciones, este enigma se convierte en una anomalía, ya que no es posible de encontrar una solución en un tiempo dado; la comunidad científica pierde la fe en el paradigma, y entonces se provoca una revolución, en la aual se abandona el viejo paradigma, reemplazándolo por otro.

La ciencia ha venido evolucionando desde muchas décadas, tal es, el estudio que ha venido realizando Bacherlard (1938), el cual ofrece una visión de la ciencia mediante aspectos históricos que han influido en la construcción del conocimiento. Surge de allí, el modelo bacherladiano, que plantea la idea de cambio cientúfico, dentro del cual el autor, muestra tres categońas que son:

Obstáculos epistemológicos: representa las formas de pensar arrizadas, las antiguas estructuras conceptuales y metodológicas; las auales obstaculizan el progreso del conocimiento científico. Basado en el animismo primitivo, el positivismo inductivo, que generó el rechazo de la metodología hipotético-deductiva.

Rupturas epistemológicas: son las formas en que el conocimiento cient́fico contradice las ideas o creencias que proceden de un conocimiento primario, intuitivo y de sentido común; tal es el caso 
de las teorías o hipótesis que muchas veces no provocan los avances de los conocimientos científicos.

Actos epistemológicos: son los mecanismos por los cuales se van superando los obstáculos epistemológicos. Así, pues, en este modelo de cambio cient́fico, las rupturas epistemológicas provocan la superación, mediante la contradicción, del modelo preestablecido de adquisición de conocimiento. Sin embargo, para Capel (1980), en muchas ocasiones lo importante en sí no es el cambio producido, sino la permanencia de antiguas concepciones. Éstas pueden subsistir ocultas bajo formas distintas, sobreviviendo a sus ońgenes y subyaciendo en muchas teorías posteriores.

En relación con las concepciones básicas bachelardianas, Gagliardi (1986) ha desarrollado, dentro del modelo constructivista, la idea de los conceptos estructurantes, se trata de conceptos que van a transformar el sistema cognitivo del alumno de tal manera que le van a permitir, de una forma coherente, adquirir nuevos conocimientos, por construcción de nuevos significados, 0 modificar los anteriores, por reconstrucción previas de los alumnos en cuanto a la identificación de los conceptos estructurantes y de sus onígenes. Estamos, pues, ante la catarsis bachelardiana.

De ahí que, investigadores que estudiaron la evolución del proceso de enseñanza-aprendizaje de la ciencia y la construcción del conocimiento indicaron, como Ander Egg (1993), que el conocimiento se basó en el método científico; Fernández (1994), señaló que para ese momento, el método cientúfico era único y universal para la producción del conocimiento; Porlán (1993) y Furió (1994), en sus estudios sobre la producción del conocimiento determinó: el Modelo Didáctico Dominante (MDD), para esa época.

A partir de lo expuesto, se examina los estudios realizados por Méndez (2007), sobre el enfoque conductista durante los siglos ya citados; y demostró: que la producción del conocimiento, se basó en un enfoque positivista, siendo este meramente objetiva; en cuanto a la enseñanza- aprendizaje, se caracterizo por ser lineal, descontextualizada, mecanicista, sin reinterpretación, sin articulación social; considerándola un modelo improductivo.

Sin embargo, la historia registra la capacidad de autoorganización de la escuela, su tendencia a re ingeniar sus postulados, a crecer frente a la entropía (Wagensberg, 1998), siendo un reflejo de ello, la transición de un modelo tradicional al tecnológico y de estos al espontaneista 0 constructivista (Porlan, 2000).

Los hallazgos de investigaciones durante estos tiempos, permiten evidenciar la construcción o configuración de un alumno pasivo, captador de información, es decir, un agente capaz de introyectar códigos lingüísticos y íconos ajenos a su campo fenomenológico o realidad individual, para de esta manera subsistir en un escenario educativo acumulador de datos o como lo denomina Freire (1974), un espacio para depositar información; haciendo de la educación una acción bancaria, donde el facilitador-reproductor emite los códigos y el discente-grabador, los guarda, sin comprender en la mayoría de los casos, la conexión, entre los datos, todo ello como resultado de una exaltación del dominio conceptual sobre las demás aristas del saber (afectivo, procedimental entre otros).

Desde esta perspectiva el aula de dase, lejos de generar conocimiento, vino a considerarse un escenario para transmitir contenidos, y las herramientas pedagógicas son catalogadas como instrumentos para activar la memorización en detrimento de la adjudicación de los significados a los estímulos admitidos sin su correspondiente digestión.

En conseauencia el enfoque positivista y conductista, ubica al docente como el centro del proceso de enseñanza-aprendizaje, y considera al alumno como una tabla rasa: hay que llenarto de conocimientos como si se levantara una pared de ladrillos (Avolio de Cols, 1998, p. 67). 
Así pues, como el mismo autor refiere, el docente conductista, está encargado de organizar la dase, identificar las respuestas que el alumno debe aprender, reconocer las capacidades del sujeto, proponer tareas y brindar recompensas a las respuestas correctas. En tal sentido se desconocen los procesos mentales del estudiante, o por lo menos, no se los toma en cuenta por la imposibilidad de hacerlos visibles; el grado de avance se mide a través de los resultados observables de sus conductas.

En cuanto al enfoque positivista, en el aprendizaje de los conocimientos para el alumno, el conductismo es útil porque le brinda un marco de contención, en todo momento reconoce daramente qué se espera de él, sin hacer el menor esfuerzo por construir la información. Sin embargo, esta utilidad se limita a las relaciones interpersonales entre docente y alumno que mantienen en el aula. Actúa eficazmente transmitiendo el carácter normativo ya que no implica una actividad de comprensión, tal el caso de la aceptación de métodos y técnicas del paradigma. En este sentido, el docente se ocupa de bajar los contenidos a los alumnos, y éstos de reproducirlos ritualmente (Perkins, 2003, p. 17) en el examen.

Desde esta perspectiva se reconoce la existencia, de la falla cuando deben intervenir procesos mentales superiores, donde el docente estimule en los estudiantes, los niveles más complejos del pensamiento, es decir, el docente conductista se enfrenta ante un obstáculo epistemológico; por lo tanto la comprensión no se logra sino a través de una explicación explícita del docente, este transmite los conocimientos y muchas veces debe caer en la limitación del lenguaje para así explicar un proceso.

\section{Influencia de la escuela positivista en el conocimiento}

Por otra parte, la escuela positivista que sustenta al método cientúfico como único y universal (Femández, 1994), se ampara en las ideas de los autores antes referidos. Este método se caracterizó principalmente, por separar las áreas disciplinarias y por fragmentar su objeto de estudio tanto como le sea posible, argumentando que la suma de las partes constituye el todo. Si bien es cierto, que tal abordaje resulta válido para muchas de las actividades científicas de laboratorio, no deja de ser menos cierto lo limitado de su aporte para solventar planteamientos que emergen de la realidad.

Las instituciones educativas tienen la responsabilidad de preparar las nuevas generaciones para convivir, compartir y cooperar en la sociedad globalizada en las que les ha tocado convivir. Uno de los caminos para resolver este vacío, es mediante el desarrollo curriaular. Para ello la complejidad de la sociedad en que vivimos, así como la del futuro, requiere de una definición del curríaulo que tome en consideración las necesidades de la sociedad, del aprendiz y de la complejidad del proceso de enseñanza aprendizaje.

El currículo es una respuesta educativa a las necesidades de la sociedad y del individuo y que requiere que el aprendiz construya significado, actitudes, valores y destrezas mediante un complejo juego entre el intelecto, los materiales educativos y las interacciones sociales; lo cual indica la importancia de establecer y de estrechar vínculos entre el mundo educativo y la sociedad (Quintana, 2005).

Es de vital importancia dejar atrás el viejo paradigma curricular que se centraba en transmitir información, datos y conocimientos en las aulas y que estos redundaba en curríaulos cargados de asignaturas, descontextualizadas y las mismas se impartía en una forma fragmentada, generando un conocimiento fraccionado en el estudiante; y como consecuencia de ello, los estudiantes no 
establecen la integración de los conocimientos adquiridos, haciéndolos significativos en una forma constructivista.

Es importante destacar, en este aporte teórico, como la influencia de la escuela positivista como enfoque para producir conocimiento, se caracterizó en una visión restringida, mecanicista y causal; descarta otro modo de producción de conocimiento, limitando el estudio de problemas complejos y obviando que "la realidad es compleja" y es "transdisciplinaria" tal como lo refiere diversos autores (Banco Mundial, 2000; Heres, 1994 y Morin, 1999); esto hace pensar en un nuevo reformulamiento global del paradigma intelectual, para entender mejor el mundo que nos rodea; de esta forma surge el paradigma sistémico. Para su aplicación se debe de partir de un reformulamiento curricular en los programas, basado en un enfoque multidisciplinario, transdisciplinario e interdisciplinario, siendo una modo de organizar los procesos de enseñanza y aprendizaje en el tratamiento de una 0 varias disciplinas, pero induyendo contenido de otras.

Desde esta perspectiva se reconoce que, la educación, el docente y su forma de impartir los conocimientos, los contenidos, el currículo, las estrategias didáctica, el aula de dase; requieren un cambio bajo los nuevos enfoques globalizantes; ubicando al proceso de enseñanza-aprendizaje en un enfoque constructivista, de teorías aúticas y teorías complejas, Porlán (1993) y Furió (1994); en ayya triada docente-alumno-aula, se encuentran en una constante reestructuración de organización, formas de impartir y producir el conocimiento.

\section{Epistemología de la didáctica de la ciencia}

Desde este punto de vista, el problema es más profundo, también pensamos que es necesario discutir desde qué postura epistemológica y desde qué modelo didáctico se imparten los contenidos. Desde hace años, se viene prestando atención a la forma en que el alumno aprende, en especial a lo que ya sabe, como determinante de lo que es capaz de aprender. Sin embargo hay otras variables que deben merecer también la atención de los investigadores en didáctica de las ciencias. Una de ellas es la naturaleza del conocimiento científico que se enseña. Otero (1989) dice: "es muy posible que los métodos, por ejemplo, hayan variado en las últimas décadas. También las ideas sobre la forma en que el alumno aprende".

Sin embargo, la estructura conceptual de la ciencia que se enseña en los cursos, se considera como algo dado e inamovible $y$ ha estado normalmente fuera del alcance e interés de la investigación educativa: no constituye una variable problemática en las reflexiones y estudios sobre didáctica de las ciencias.

El modelo de enseñanza - aprendizaje en la didáctica de la ciencia, debe estar orientado a promover un cambio en la metodología, acompañado de una profundización en el análisis de la estructura conceptual de la disciplina. El problema de la enseñanza de las ciencias, reside en reflexionar sobre qué contenidos enseñar. Es decir, encontrar una manera de transformar el contenido "privado" en "público" (Otero, 1989).

Por otra parte, el problema de la enseñanza y aprendizaje de las ciencias es, en síntesis, un problema de "transposición didáctica". Para el desarrollo de esta investigación, es importante contar con un aporte desde la epistemología de manera tal que sea posible encontrar un paralelismo entre la creación del conocimiento y su construcción por parte del alumno. Teniendo en cuenta la función de la ciencia propuesta por (Alexaindre y Giere del Carmen y otros, 1997) ambos hacen referencia a la existencia de modelos explicativos que los alumnos deberán ir incorporando paulatinamente a través del proceso de enseñanza y aprendizaje. 
A partir de lo expuesto se pueden examinar, los estudios sobre la tayectoria cognitiva que siguen los alumnos en una dase, bajo la orientación de la epistemología constructivista. Más precisamente, es necesario conocer el itinerario que siguen los alumnos para superar ciertos obstáculos epistemológicos, semánticos y didácticos. Pero se hace necesario de conocer el proceso mental que permita establecer conexiones y activar el conocimiento previamente adquirido; iniaiando con la integración auriaular que permita facilitar e integrar los niveles complejos del conocimiento con el concepto y el tema que se estudia. Una de las forma es mediante la multidisciplinariedad y la interdisciplinariedad.

La multidisciplinariedad es actualmente es considerada un enfoque, que permite organizar los procesos de enseñanza y aprendizaje centrado en el tratamiento de uno o varios temas desde la perspectiva o el lente o el lente de una disciplina, pero induyendo el contenidos de otras, en este caso el estudiante es el que establece las relaciones necesarias entre las diversas aproximaciones de las disciplinas para integrar el conocimiento (Drake, 1993).

\section{Recomendaciones}

En esta discursividad que realizo con estos enfoques, deseo destacar a modo de recomendación, que la integración curricular representa la altemativa, para evitar reducir el auríaulo; provee de profundidad a los procesos de enseñanza y aprendizaje; involucra al estudiante en un proceso de aprendizaje más activo; estimula los niveles más complejos del pensamiento llevándolo a la integridad de los conocimientos de la ciencia.

En el caso particular, en Venezuela se ha asistido a una recurrente reestructuración organizativa del diseño curricular, desde los años 1996 que, en el caso de la educación, ha llevado, por ejemplo, a la eliminación y creación de dependencias en el Ministerio de Educación; a la integración de la Universidad Pedagógica Experimental Liberatdor (UPEL); a las periódicas reformas curriculares; como es la transformación y Modemización del Diseño Curricular UPEL; el cual propone las innovaciones auriaulares en materia de integración, las competencias del egresado, entre otros cambios, que, justificados o no, se identifican con ese tipo de pensamiento de ciudadano que se requiere en la sociedad actual y del mundo en tiempos de globalización.

La búsqueda sistemática y rigurosa de respuestas a las inquietudes de la investigadora, contribuirán, al desarrollo de un modelo educativo socio cognitivo, producto de una investigación cualitativa; de la dinámica de aula, entre la epistemología - axiología - aprendizaje centrado en un paradigma de desarrollo integral, sobre la base de una didáctica científico-ática; estos hallazgos nos aproximarán a las verdades provisionales como un intento de resolver el vacío teórico o epistemológico.

\section{Bibliografia}

Ander-Egg, E. (1993). Interdisciplinariedad en educación. Buenos Aires: Magisterio el Río de la plata.

Del Carmen, L., y otros (1997). La enseñanza y el aprendizaje de las ciencias de la naturaleza en la educación secundaria. Universitat de Barcelona, ICE / HORSORI.

Furió, C. (1994). Tendencias actuales en la formación del profesorado de ciencias. Enseñanza de las ciencias, 12 (2),188-199.

Porlán, R. (1993). Constructivismo y escuela. Hacia un modelo de enseñanza-aprendizaje basado en la investigación. Sevilla: Didáctica Editora. 